Should the child be examined and where?

The child should be examined but not without the knowledge and agreement of a parent (or the order of a court). Mothers of preadolescent children should always be invited to be present, except in the most exceptional circumstances. Adolescent patients should be asked whether they wish a parent to be present.

It is usually counterproductive to examine a resistant child, and if his or her cooperation cannot be obtained the examination should be deferred unless there are urgent medical reasons to proceed.

The child should be examined as soon as optimal arrangements can be made. Few children require urgent examination.

Repetitive examination is usually abusive and should be avoided.

The examination should be conducted in absolute privacy and in an environment where the child can be comfortable - not behind screens in open wards or in police stations.

There should be adequate equipment for any necessary diagnostic tests. Recording and photographic facilities are an advantage but their value is outweighed if they cause distress to the child or mean that another examination has to be conducted.

\section{Who should examine?}

A person with skill in paediatric examination who is familiar with normal genital and anal appearances of children should conduct the examination. When physical abnormalities are expected from the history a forensic physician should be invited to examine or to be present during the examination so that it need not be repeated and a second opinion is available in doubtful cases.

\section{Who should be present?}

The only people present at the examination should be the child, his or her parent, and the examiner(s) - no one else-except, with the agreement of the parents, an occasional observer in training.

\section{What about acute sexual abuse?}

When abuse is thought to have been recent (within 72 hours) or there is serious genital injury forensic evidence must not be compromised. Examination should be deferred, if consistent with safety, until a forensic physician can be present. Nobody should remove clothing or attempt to clean or bath the child. Junior medical staff should not examine suspected victims unless the child urgently needs medical attention.

For Debate

\title{
Patient preferences and randomised clinical trials
}

\author{
C R Brewin, C Bradley
}

Allocating patients to different treatments by randomisation in a controlled trial is now accepted almost without question in accounts of trial design.' Randomisation may reasonably be supposed to play a large part in evaluating proposed studies for grant support. The virtue of randomisation is that it reduces some types of systematic error that may interfere with the interpretation of the results of a trial. Allocating patients to treatments in a systematic non-randomised way may introduce bias which destroys comparability. We argue here that despite this advantage random allocation is not always suitable. Though patients play an active part in the outcome of all treatments, we suggest that clinical trials in which they are required to sustain an effortful and demanding role and those in which they are likely to have strong preferences for one treatment need to be considered and conducted differently.

MRC Social Psychiatry

Unit, Institute of

Psychiatry, London

SE5 8AF

C R Brewin, PHD, research

clinical psychologist

Department of Psychology, University of Sheffield

C Bradley, PHD, lecturer in

psychology applied to medicine

Correspondence to:

Dr Brewin.

Br.Med. 7 1989;299:313-5 cognitive treatments for anxiety and depression; and deinstitutionalisation programmes for chronically mentally ill and mentally handicapped patients.

The success of many, but particularly participative, interventions depends further on the patients' perceptions of their suitability and on patients' willingness to help to make them succeed. ${ }^{23}$ If effectiveness is evaluated after random administration to patients who may or may not desire the treatment it will be difficult to distinguish between a treatment that failed because it was not inherently effective and one that failed because it was not targeted towards patients who understood why that treatment was given or who were suitably motivated. Traditionally, motivation was seen as a characteristic of the patient which, it was assumed, did not change with the nature of the treatment offered. If this is the case then conventional randomisation ensures that different groups contain equal numbers of those who are "well" motivated and "poorly" motivated. With participative interventions, however, it seems more realistic to view motivation in terms of the "fit" between the nature of the treatment and the patient's wishes and perceptions. If an attempt is not made to achieve a good fit misleading underestimates of effectiveness are likely to result.

\section{Information}

Consider the case where the investigator wishes to compare two treatments of which at least one is participative. If patients are randomly allocated to treatments the investigator may draw conclusions 
about their relative efficacy only if it is assumed that motivational factors are equally important or unimportant in the two groups. If patients are not informed that they are taking part in a trial or about the side effects, discomfort, inconvenience, disruption to routine, and the length of time and amount of effort entailed for both arms of the trial then it will not be possible to elicit whether the assumption is justified. Thus for trials of this kind it is vital to give patients sufficient information.

It may be argued that matching on motivational variables can be achieved by providing information and then including only patients who are fully informed and who are then willing to accept random allocation. In practice patients may still have strong preferences for one form of treatment and may, for example, agree to participate because this is the only way that they stand a chance of receiving a new or experimental treatment $t^{4}$ or because they are hoping to be allocated to a particular and preferred group. Thus despite having full information and giving consent patients may still find themselves allocated to non-preferred treatments, which lowers their motivation to make the treatment work. Biased estimates of effectiveness would then be apt to occur.

\section{The alternative ... design is to optimise motivation by ascertaining patients' preferences.}

Random allocation may work in participative trials if treatments are viewed as similar by patients who have no preferences and the investigator wishes to test a difference in some component that is assumed to be central to the mode of action of the treatment and thus crucial to its success. Many trials of participative interventions, however, are not of this kind. There are many ways of helping cardiac patients to recover, of helping people with insulin dependent diabetes to control their blood glucose concentrations, and of encouraging patients to manage symptoms of anxiety and depression, just as there is a variety of possible community settings into which mentally ill or handicapped patients may be discharged. The aim of each intervention is clear. The issue is how it can best be achieved. Often participative treatments educate and motivate patients to manage or adapt to their condition without assuming that there is only one correct way. For example, in cognitive therapy for depression ${ }^{5}$ the heterogeneity of patients is explicitly recognised and specific techniques are prescribed and adapted to individual need.

\section{Explanatory and pragmatic attitudes}

Our argument is related to the distinction between explanatory and pragmatic attitudes to the design and conduct of clinical trials. ${ }^{6}$ In explanatory trials the aim is to reduce the possible differences between the two arms to a minimum to be able to pinpoint what makes one treatment better or worse. In pragmatic trials the objective is to discover which of two different "packages" of management gives better results even though the importance of the individual components of the package cannot necessarily be identified. Most treatments are complex, consisting of a mix of supposedly active components with contextual factors such as, for example, the way the treatment is given, side effects and their management, and auxiliary care. In explanatory trials the contextual factors are "equalised" by randomisation and often also by imposing artificial restraints on their presence. In pragmatic trials the contextual factors are optimised, so approximating to actual practice. We suggest that with participative interventions the contextual factors include motivational concerns such as the patient's belief in and preference for one kind of treatment over another. Pragmatic designs which capitalise on rather than ignore the differences between patients and ask what the most suitable intervention is under optimal motivational conditions are therefore most suitable to test participative interventions. For example, we could fit patients to treatments by asking them to select the one that in their opinion suits them best. We illustrate this alternative to random allocation by considering research into two different illnesses-introducing insulin infusion pumps in the self management of diabetes mellitus and introducing counselling after surgery for women with breast cancer.

\section{Choosing the most suitable treatment}

SELF MANAGEMENT OF DIABETES MELLITUS

Continuous subcutaneous infusion of insulin is a relatively recent development in managing diabetes. The question about its possible value is pragmatic: Can its use be recommended in preference to or in addition to other treatment regimens? Not all patients with poorly controlled blood glucose concentrations find the technique attractive - for example, because of the need to wear a pump strapped to the body. When given a choice between this and more familiar methods many patients do not select this method. ${ }^{7}$ Therefore with random allocation there will always be a risk that the groups will not be matched for motivational variables, and, strictly speaking, random allocation should be done only for a group of patients who at the outset have no preference for either the new or the old treatment. The evidence suggests that it would be hard to find sufficient patients who were unconcerned about the method of insulin delivery and that either a trial would therefore not be possible or the subjects would be unrepresentative. Furthermore, randomising patients to a treatment they do not want will increase the dropout rate and thus limit the conclusions that can be drawn. ${ }^{4}$

In the most suitable design, therefore, motivational factors would be optimised by letting patients select their preferred method. This was done in the Sheffield feasibility study, ${ }^{7}$ when after an introductory explanation about the options patients chose continuous subcutaneous insulin, or a conventional self injection regimen, or an intensified version of the second. By giving these choices the investigators were able to include almost an entire clinic population in the study and thus achieve a high degree of representation. Because the study was designed in this way the results cannot tell us about the relative efficacy of the three treatments for any patients with diabetes requiring insulin, but they can tell us whether a new technique is a viable option for patients who choose to use it and whether it would be good for them because their blood glucose concentrations would be better controlled compared with baseline measures. During this study the preferences of patients were a focus of interest, and the factors that determined choice were explored. As a result, recommendations were made for more suitable education to enable patients to judge which treatment would best suit their needs and priorities. ${ }^{89}$

\section{COUNSELLING AFTER SURGERY}

The ethical issues concerned in a randomised trial of counselling versus no counselling for patients undergoing mastectomy when entry into the trial was not subject to informed consent were raised some time ago ${ }^{10}$ and again recently. ${ }^{112}$ There is a need to 
know if counselling is effective, but randomisation implies that counselling must be shown to be better than no counselling regardless of whether patients wish to be counselled. It would be easy to imagine that the two groups (counselled and not counselled) contain equal numbers of patients who would like to be counselled and those who wish to get on with their lives without counselling. One group is then counselled and the other is not. In each the patients whose wishes are met demonstrate better psychological adjustment than those whose wishes are contravened, and the overall adjustment of the two groups is about the same. In such a study the conclusion that is likely to be reached is that the efficacy of counselling has not been proved, though an internal analysis taking into account the wishes of the patients would lead to a different conclusion.

Irrespective of the possibility of getting a "wrong" answer by failing to consider attitudes, a randomised trial of counselling against no counselling without an attempt to ascertain the patients' wishes is logical only if one of two assumptions holds. One is that counselling will be effective whether or not the patient wants it and participates in it-a premise that few counsellors would claim to be justified. The other is that patients' preferences for or against are homogeneous. They are $(a)$ all against it, in which case the trial is probably a waste of time, or $(b)$ uniformly indifferent to whether they are counselled or not, which seems unlikely, or $(c)$ all for it, which is perhaps the most plausible form of homogeneity. Should the last be true, however, then it is scarcely justifiable except on grounds of resources for withholding it from some patients in the interests of a clinical trial.

The alternative and perhaps more suitable design is to optimise motivation by ascertaining patients' preferences. Patients who have no preference are then randomly allocated to counselling (group A) or no counselling (group B). Two further groups would include patients who chose counselling (group C) and those who did not wish it (group D). These groups would give an estimate of the value of the intervention per se $(\mathrm{A} v \mathrm{~B})$ and of the additional influence of motivational factors $(\mathrm{A} v \mathrm{C}$ and $\mathrm{B} v \mathrm{D})$ ). If group $\mathrm{C}$ did much better than group $\mathrm{D}$, or vice versa, then it might be necessary to ask if the outcome was a result of self selection bias or if it represented a genuine treatment effect. The implications for providing care might include advocating one form of care despite an initial lack of enthusiasm from the patient. We contend, however, that such a case could be made only after a study of the kind that we describe was undertaken.

\section{Conclusion}

We argue that simple randomised controlled trials may not always be suitable to evaluate participative interventions. Though the purpose of randomisation is to ensure equal distribution of all factors, including motivational ones, among the two or more arms of a trial, this will not necessarily be achieved, particularly when the patient prefers one of the treatments. The pragmatic question of which treatment is more useful under what are judged to be optimal conditions of motivation should be considered as a legitimate alternative to the explanatory, but sometimes unrealistic, question of which is the best treatment per se. Investigators must be clear which class of question each kind of trial can and cannot answer. Problems of interpretation are bound to arise when trials of participative treatments are designed as though they are drug trials without considering the different psychological processes concerned in each.

We thank Dr G Dunn and Professor J K Wing for comments on an earlier draft of this paper.

1 Pocock SJ. Clinical trials: a practical approach. Chichester: Wiley, 1983.

2 Brewin CR. Cognitive theories of motivation. In: Eysenck HJ, Martin I, eds. Theoretical foundations of behavior therapy. New York: Plenum, 1987

3 Brewin CR. Cognitive foundations of clinical psychologv. London: Erlbaum, 1988

4 Bradley C. Clinical trials: time for a paradigm shift? Diabetic Med 1988;5: 107-9.

Beck AT, Rush AJ, Shaw BF, Emery G. Cognitive therapy of depression. New York: Wiley, 1979.

6 Schwartz D, Lellouch J. Explanatory and pragmatic attitudes in clinical trials. f Chronic Dis 1967;20:637-48.

7 Knight G, Boulton AJM, Drury J, et al. A feasibility study of the use of continuous subcutaneous insulin infusion in a diabetic clinic: patients' choice of treatment. Diahetic Med 1984;1:267-72.

8 Bradley C, Gamsu DS, Moses JL, et al. The use of diabetes-specific perceived Bradley C, Gamsu DS, Moses JL, et al. The use of diabetes-specific perceived
control and health belief measures to predict treatment choice and efficacy in a control and health belief measures to predict treatment choice and efficacy in a
feasibility study of continuous subcutaneous insulin infusion pumps. feasibility study of continuous sub
Psychology and Health 1987;1:133-46.

Psychology and Health 1987;1:133-46.
9 Bradley C, Gamsu DS, Knight G, Boulton AJM, Ward JD. Predicting risk of Bradley C, Gamsu DS, Knight $\mathrm{G}$, Boulton $\mathrm{AJM}$, Ward JD. Predicting risk of
diabetic ketoacidosis in patients using continuous subcutaneous insulin infusion. BrMed f 1986;293:242-3.

10 Thomas E. Informed consent. Lancet 1986;ii: 1280

11 Raphael A. How doctors' secret trials abused me. Observer 1988 Oct 9:12 (col 1).

12 Baum M. I didn't abuse my patients. Observer 1988 (Ot 16:8 ( $\mathrm{col} 1)$.

(Accepted 18 May 1989)

\section{MATERIA PARAMEDICA}

\section{The toss of a coin: how to advance your career}

I have known of appointments committees that were equally divided in the members' preference between two suitable candidates. A vacancy has to be filled; there is no time to reconvene another committee, and either candidate is acceptable. The toss of a coin is then a quick way of creating a much needed bias. This may seem a bit hard on the loser, but who can tell where one's luck lies? The following story shows an unusual twist on this unusual theme.

In 1930 the London County Council (later the GLC) took over 89 backward, underfunded hospitals from the Metropolitan Asylums Board and the Boards of Guardians. Sir Frederick Menzies was in charge, and the immense task of upgrading the hospitals and equipment and staff was completed within three years. Among the new staff were two able young surgeons. One day, in the course of their duties, a patient had to undergo amputation of a finger. The surgeons tossed a coin to decide who would operate and who would give the anaesthetic. In those days, despite improved staffing in municipal hospitals, one had to be a jack of all trades; anaesthetists were a rarity. The coin was tossed, and the surgeon whom I shall call KN gave the anaesthetic, while DR amputated the finger.
Unfortunately, he removed the wrong finger. The authorities at County Hall were not amused. KN and DR were candidates for the post of medical superintendent.

The medical superintendent of a London County Council hospital was provided with a large house, rent and rates free, with heating, gas, electricity, and laundry thrown in for good measure. In due course a vacancy was advertised and $\mathrm{KN}$ got the job. It was my privilege to work under him: an able administrator, a gentle disciplinarian, and scrupulously fair. He ran the course and retired at age 65 .

But what happened to DR? County Hall decided that he should wait. Eventually he was made superintendent at a hospital in north London. Now, it was the London County Council's policy to create specialised units at various general hospitals, and a goitre clinic was established at the north London place, later renamed the Thyroid Clinic. Here DR worked with the best thyroid surgeons of the day, Cecil Joll, Thomas Dunhill, and Geoffrey Keynes; and, on the medical side, with Raymond Greene. DR's status as a surgeon on the thyroid and thymus (for myasthenia gravis) was greatly enhanced and he was created CBE. So much, then, for the toss of a cOIn. - BERNARD J FREEDMAN 